\title{
Effect of penetrants on the aramid Nomex
}

\author{
ANJANA JAIN and KALYANI VIJAYAN* \\ Materials Science Division, National Aerospace Laboratories, Bangalore 560 017, India
}

MS received 16 December 1999; revised 21 February 2000

\begin{abstract}
The response of Nomex sheets to some penetrants has been analyzed. The process of moisture uptake depends on the relative humidity (RH) of the ambient atmosphere and the initial characteristics of the polymer. In the case of common laboratory solvents, the uptake by the polymer shows an inverse dependence on the molar volume of the former. Although the calendered and the uncalendered Nomex sheets exhibit an overall similarity in their response to various penetrants, quantitative comparison reveals distinct differences.
\end{abstract}

Keywords. Nomex; relative humidity; penetrants.

\section{Introduction}

As is well known, Nomex is an aramid made up of poly $(m$-phenylene isophthalamide) or (PMIA) (Tadokoro 1979), the structural formula of which is shown below. Sheets of Nomex find applications in different types of environments, which include wet conditions too. This communication reports quantitative data concerning the

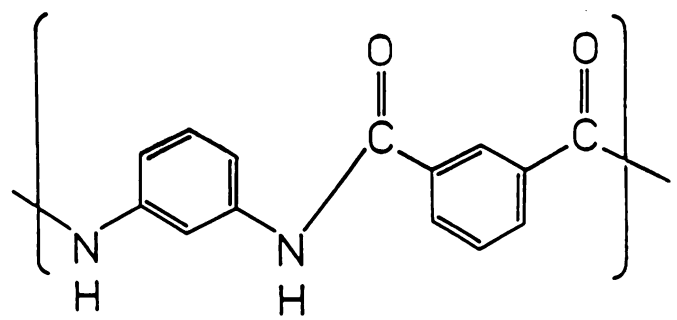

exposure of two types of Nomex sheets viz. the uncalendered 411 and the calendered 410 varieties, to moisture and other common laboratory solvents. In the former case, the influence of relative humidity $(\mathrm{RH})$ of ambient atmosphere as well as the initial structural characteristics of the sheets have been examined. The present study also aims to decipher differences in the initial characteristics of the two types of sheets and correlate differences if any, with their behaviour. Detailed investigations have been carried out in the past on the aramid Kevlar (Shubha 1989; Parimala 1991; Vijayan et al 1991, 1996; Shubha et al 1993; Iyer 1999), which is the para substituted isomer of Nomex (Kwolek 1972, 1974). In this context, it was also of inte-

\footnotetext{
*Author for correspondence
}

rest to compare the behaviour of the two isomers. It must be mentioned here that the present study on the aramids is expected to provide data which are specially relevant to the users of these materials.

\section{Experimental}

The samples used in this study were $\approx 10$ mil thick sheets made commercially available by DuPont. For each experiment, a piece of dimension $\approx 30 \times 30$ sqmm was used. Initial characterization of the samples was carried out by X-ray diffraction methods and scanning electron microscopy. Wide angle X-ray diffraction patterns were recorded with $\mathrm{CuK}_{\alpha}$ radiation using a Philips powder diffractometer with a proportional counter and a graphite monochromator in the diffracted beam. Values of crystallinities were estimated from the respective integrated intensities of diffraction profiles. Micrographs were recorded from gold coated samples using a Jeol scanning electron microscope. Employing the physical dimensions of the sheets and the weight, the respective densities were estimated. The loss in weight incurred on exposing the sheets to $150^{\circ} \mathrm{C}$ for $1 \mathrm{~h}$ provided an estimate of the equilibrium moisture content.

The effect of RH on the process of moisture uptake was analysed using a Sartorius analytical balance as the humidity chamber. Using a judicious combination of calcium chloride and water contained in separate glass holders, the humidity within the chamber was monitored to 20, 40 and $60 \%$, respectively. The $\mathrm{RH}$ within the chamber stabilized to the required value within $\approx 1.5 \mathrm{~h}$ and thereafter remained stable within $\pm 1 \%$, over a period of $6 \mathrm{~h}$. The fluctuations increased to $\pm 3 \%$, within a span of $24 \mathrm{~h}$.

Samples with an initial weight $w_{\mathrm{s}}$, were dried for $16 \mathrm{~h}$ at $150^{\circ} \mathrm{C}$, cooled in a dessicator containing silica gel and then transferred to the humidity chamber. Changes in 
weight were attributed entirely to the entry of moisture from the ambient atmosphere into the sample. For each of the dry samples, changes in weight were followed closely for the first $6 \mathrm{~h}$. Subsequently, the gain in weight after $24 \mathrm{~h}$ of exposure, $\Delta w_{24}$, has been noted. The percentage increase in weight was estimated as $\left(\Delta w_{1} / w_{\mathrm{d}} \times 100\right)$.

Here,

$$
\Delta w_{1}=w(t)-w_{\mathrm{d}},
$$

$w_{\mathrm{d}}$ and $w(t)$ represent the weights of the sample prior to and after $t$ min of exposure to a chosen humidity, respectively.

In the case of liquid penetrants, dry sheets were cooled and dropped into the respective liquids of interest which included formaldehyde, methanol, acetone, butanol, carbon tetrachloride, benzene, chlorobenzene, acetophenone, mesitylene and water. After $8 \mathrm{~h}$ of soaking in the solvent, the sample was picked with a tweezer, dried between filter papers and weighed $\left(w_{\mathrm{s}}^{1}\right)$ immediately. The difference in weight, $w_{\mathrm{s}}^{1}-w_{\mathrm{d}}$, represents the uptake of solvent by the dry sheet.

To compare the response of Nomex to liquids and their vapours, dry sheets were exposed to the respective vapours of the above mentioned liquids, using a sealed bell jar. The weight of the sheet after $8 \mathrm{~h}$ of exposure to the vapours, $w_{\mathrm{s}}^{\mathrm{v}}$, was estimated.

\section{Results and discussion}

Preliminary X-ray characterization showed that the calendered 410 sheets were twice as crystalline as the uncalendered sheets (figure 1). The density values, $\rho_{410}=$

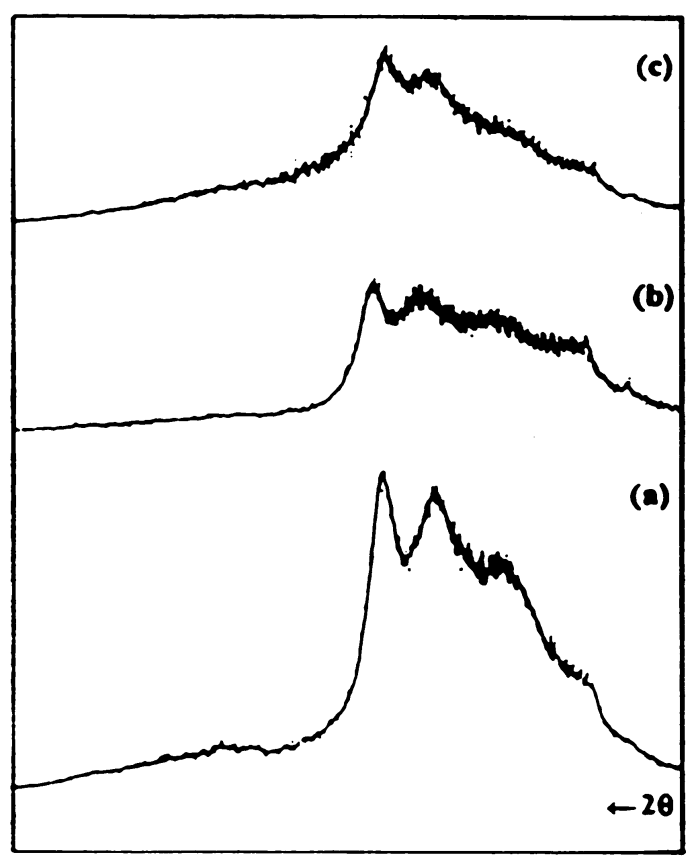

Figure 1. $\delta$ and $\lambda$ comparison of X-ray diffraction patterns from (a) 410, (b) 411 and (c) 411 saturated with water.
$0.98 \mathrm{~g} \cdot \mathrm{cm}^{-3}$ and $\rho_{411}=0.33 \mathrm{~g} \cdot \mathrm{cm}^{-3}$, indicate that 410 is approximately three times more crystalline than 411 . The slight discrepancy between the ratios of crystallinities derived from X-ray data and the density value is understandable because the density value represents the entire sample whereas the X-ray diffraction intensities pertain only to the crystalline fraction of the sample. As indicated by the scanning electron micrographs (figure 2), the packing of fibres is less efficient in 411 than in 410 . These data suggest that inherently, the 411 sheets are endowed with more room to retain water or any other extraneous molecule, than the 410 sheets. Values of the equilibrium moisture content viz. 5.6(3) and 7.5(8) wt.\% obtained for the 410 and 411 sheets, respectively provide support for such a difference in retention. The equilibrium
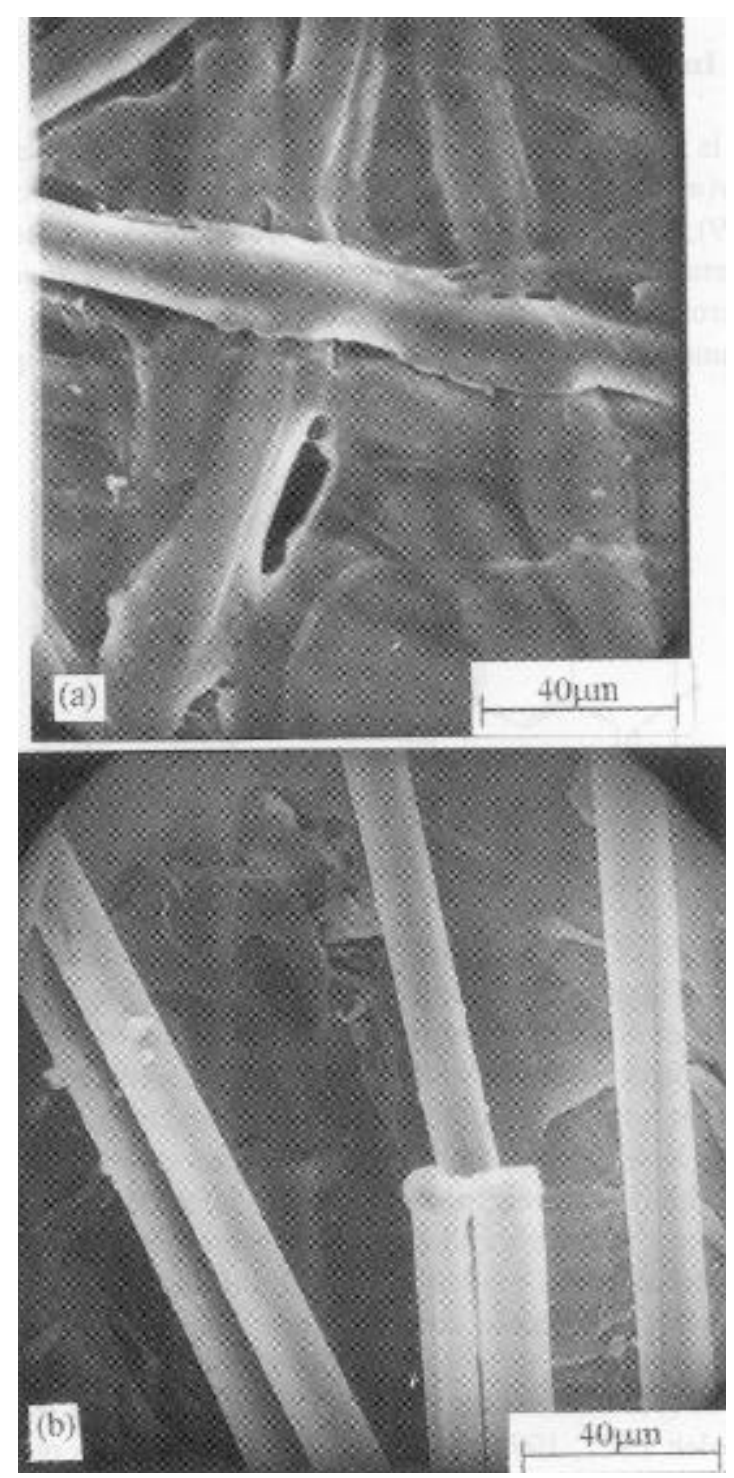

Figure 2. Scanning electron micrographs from (a) 410 and (b) 411 . 
moisture contents obtained for the sheets used in this study are slightly higher than the values reported in literature viz. 4-5 wt.\% (Carter 1971; DuPont 1981).

Figure 3 presents the sorption curves. Each data point is an average from two measurements and the standard deviations range from 0.5 to $1.8 \%$ of the values. Figure 3 shows that for both the varieties, at any point of time, the rate of moisture uptake increases with the $\mathrm{RH}$ value and that the uncalendered sheets retain significantly more amount of moisture than the calendered variety. The latter feature could be attributed to the lower crystallinity as well as the mode of arrangement of fibres in the 411 sheets. These features are similar to the observations on Kevlar fibres and unoriented PPTA or poly $(p$-phenylene terephthalamide), the polymer of which Kevlar is made of, where it was shown that increase in the RH value, reduction in crystallinity and molecular misalignment enhanced the moisture uptake (Parimala 1991; Shubha et al 1993). Figure 3 shows that sorption by Nomex is essentially a two-stage process whose feature is similar to that observed in hydrophilic polymers like wool (Downes and Mackay 1958), cellulose (Newns 1959) and nylons (Asada and Onogi 1963).

Sorption can be associated with two types of waterpolymer interactions viz. (i) the water molecules are

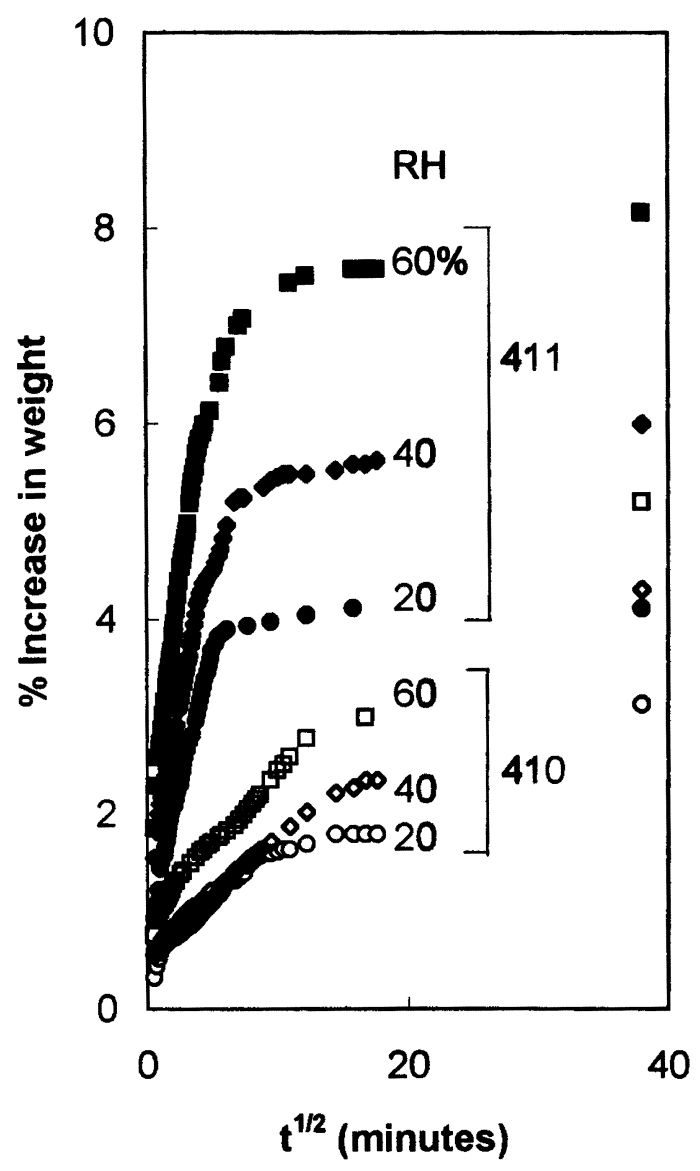

Figure 3. Sorption curves for various $\mathrm{RH}$ values. bound to the polymer by, say, formation of hydrogen bonds and (ii) the water molecules are loosely bound or clustered. In the former case of bound water molecules, two possibilities exist, viz. (a) the water molecules enter the unit cell of the crystal lattice and bind to the amide $\mathrm{CO}$ and $\mathrm{NH}$ groups of the polymer and/or (b) the water molecules do not gain entry into the unit cell and they bind themselves only to the free $\mathrm{CO}$ and $\mathrm{NH}$ groups on the surface of the crystallites/fibre. If the water molecules entered the unit cell, the entry should manifest as changes in the unit cell constants as well as the intensity distribution in the X-ray diffraction pattern. Examination of the patterns recorded from samples saturated with water (figure 1), did not provide any evidence for the introduction of such changes. X-ray data thus suggest that the sorbed water molecules do not enter the unit cell and that their binding is likely to be confined to the $\mathrm{CO}$ and $\mathrm{NH}$ groups present outside the crystal lattice, say on the surface of the crystallites. It must be pointed out that in the case of Kevlar also the water molecules do not enter the crystal lattice (Vijayan et al 1991). In the case of both the aramids, the crystal structures appear sufficiently rigid that despite the presence of the hydrophilic amide groups in the molecules, the water molecules do not gain entry into the unit cell. The second mode of interaction, viz. clustering of water molecules appears to be easily facilitated by the inherent macro structural characteristics viz. the gaps between the staple fibres described earlier (figure 2).

Figure 4 records the results concerning other liquid penetrants. Here, the increase in weight has been con-

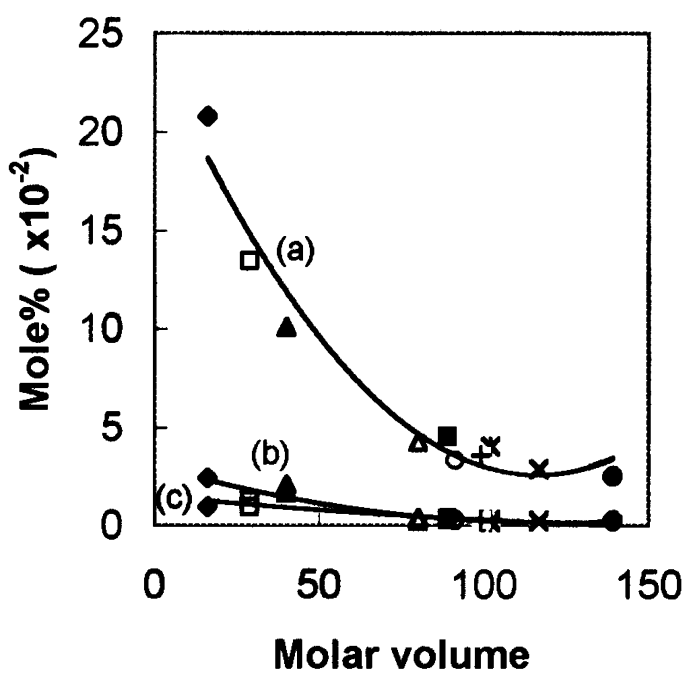

Figure 4. Mole \% versus molar volume of penetrants (a) 411 in liquids, (b) 410 in liquids and (c) 411 exposed to vapours. $((\diamond)$ water, $(\square)$ formaldehyde, $(\boldsymbol{\Delta})$ methanol, $(\Delta)$ acetone, $(\boldsymbol{\square})$ benzene, $(O)$ butanol, $(+)$ carbon tetrachloride, $(*)$ chlorobenzene, $(\boldsymbol{*})$ acetophenone and $(\bullet)$ mesitylene). Equations representing the nonlinear uptakes are (a) $y=0.0016 \quad x^{2}-0.37$ $x+24 \cdot 30$; (b) $y=0.0020 x^{2}-0.05 x+3 \cdot 06$; (c) $y=0.0001 x^{2}-$ $0.02 x+1.55$ 
verted into the corresponding mole per cent values. The mole per cent exhibits a nonlinear, inverse dependence on the molar volume of the penetrant molecules. Equations representing this behaviour are also included in figure 4. Similar inverse dependence on the molar volume of penetrants has been observed in the case of Kevlar fibres (Vijayan et al 1996; Iyer 1999) and some polyurethane membranes also (Aithal and Aminabhavi 1990).

Comparison of the data in figure 4 shows that on an average, the 411 sheets retain ten times more of solvents than the 410 sheets. For all the solvents used in this study, 411 retains more than $100 \mathrm{wt} . \%$, the lowest value being 104 wt.\%, for butanol. Despite retention of such large quantities, the sheets did not exhibit any uniform swelling. The surface, however, included several localized, blisterlike swellings. The strikingly increased uptake by the 411 sheets could perhaps be due to differences in treatment given during manufacture the details of which are, however, not known. Figure 4 also includes the data concerning exposure of the 411 sheets to vapours. The uptake of vapours is conspicuously much less than that from liquids.

Does the retention of penetrants, whether it is from liquid or vapour, cause any permanent damage to the sheets? Examination of the X-ray patterns and the weight of samples one week after exposure showed that the retention of penetrants by Nomex was a temporary effect and no permanent damage was incurred. It appears therefore that Nomex sheets which are used in wet environments of the type considered in the present study, can be reused.

In contrast with the penetrants described thus far, DMSO was found to be hostile to Nomex, causing separation of the staple fibres and lending a rather ruffled appearance to the sheets. It must be pointed out that in the case of Kevlar fibres also, DMSO had a distinct effect viz. the uptake of DMSO by Kevlar was conspicuously high (Vijayan et al 1996; Iyer 1999) and this feature was attributed to possible interactions between the polymer and the reactive functional groups in DMSO. In the case of Nomex, it appears that such interactions are perhaps more severe to the extent of causing physical damage to the sheets.

\section{Conclusion}

The calendered 410 sheets are more crystalline than the uncalendered 411 variety. The latter retains more of moisture and common laboratory solvents than the former. In both 410 and 411, the moisture uptake is dependent on the relative humidity of the ambient atmosphere. The water molecules from moisture uptake do not appear to enter the unit cell of the crystal lattice. The uptake of liquid penetrants exhibits an inverse proportionality on the molar volume of the solvents.

\section{Acknowledgements}

The authors wish to thank the Aeronautical Research and Development Board of India for the sanction of a project which enabled this study. One of us (AJ) thanks Naltech Pvt Ltd for the award of a contract post. The authors acknowledge the help given by $\mathrm{Dr} \mathrm{T}$ A Bhaskaran in recording the scanning electron micrographs.

\section{References}

Aithal U and Aminabhavi T M 1990 Indian J. Tech. 28592

Asada T and Onogi S 1963 J. Coll. Sci. 18784

Carter M E 1971 in Essential fibre chemistry (New York: Marcel Dekker Inc.) p. 110

Downes J G and Mackay B H 1958 J. Polym. Sci. 2845

DuPont 1981 Technical Information Bulletin NX-16

Iyer R V 1999 X-ray and related studies on polymeric materials $\mathrm{Ph} . \mathrm{D}$. thesis, Bangalore University, Bangalore

Kwolek S L 1972 US Pat. 3,671, 542

Kwolek S L 1974 US Pat. 3,819, 587

Newns A C 1959 J. Polym. Sci. 41425

Parimala H V 1991 Some investigations on the properties and behaviour of Kevlar fibres, M. Phil. dissertation, Mangalore University, Mangalore

Shubha M 1989 X-ray investigations on the aramid poly (p-phenylene terephthalamide), M. Phil. dissertation, Mangalore University, Mangalore

Shubha M, Parimala H V and Vijayan K 1993 J. Mater. Sci. Lett. 1260

Tadokoro H 1979 Structure of crystalline polymers (New York: John Wiley \& Sons) p. 396

Vijayan K, Parimala H V and Shubha M 1991 Polymer science-contemporary themes (ed.) S Sivaram (New Delhi: Tata McGraw Hill) p. 666

Vijayan K, Iyer R V and Jain A 1996 Indian J. Chem. Tech. 3 164 